\title{
Effects of miR-124-3p regulation of the p38MAPK signaling pathway via MEKK3 on apoptosis and proliferation of macrophages in mice with coronary atherosclerosis
}

\author{
Chuannan Zhai ${ }^{1,2, A-E}$, Hongliang Cong ${ }^{1,2, E, F}$, Kai Hou ${ }^{1,2, A-C}$, Yuecheng $\mathrm{Hu}^{2, A, B}$, \\ Jingxia Zhang ${ }^{2, A, B}$, Yingyi Zhang ${ }^{2, A, B}$, Ying Zhang ${ }^{2, A, B}$, Hong Zhang ${ }^{2, A, B}$ \\ ${ }^{1}$ School of Medicine, Nankai University, Tianjin, China \\ ${ }^{2}$ Department of Cardiology, Tianjin Chest Hospital, China \\ A - research concept and design; $\mathrm{B}$ - collection and/or assembly of data; $\mathrm{C}$ - data analysis and interpretation; \\ $D$ - writing the article; $E$ - critical revision of the article; $F$ - final approval of the article
}

Address for correspondence

Hongliang Cong

E-mail: hl_cong56uj@163.com

Funding sources

None declared

Conflict of interest

None declared

Received on January 12, 2020

Reviewed on February 19, 2020

Accepted on May 1,2020

Published online on August 4, 2020

Cite as

Zhai C, Cong H, Hou K, et al. Effects of miR-124-3p regulation of the p38MAPK signaling pathway via MEKK3 on apoptosis and proliferation of macrophages in mice with coronary atherosclerosis. Adv Clin Exp Med. 2020;29(7):803-812. doi:10.17219/acem/121926

DOI

10.17219/acem/121926

Copyright

Copyright by Author(s)

This is an article distributed under the terms of the

Creative Commons Attribution 3.0 Unported (CC BY 3.0)

(https://creativecommons.org/licenses/by/3.0/)

\begin{abstract}
Background. Atherosclerosis (AS) is the main cause of myocardial infarction and stroke. Macrophage apoptosis in the early stages can attenuate lesions, while in the late stage it is associated with AS plaque rupture.

Objectives. To explore the effects of miR-124-3p regulation of the p38MAPK signaling pathway via the MEKK3 gene on the apoptosis and proliferation of macrophages in mice with coronary AS.

Material and methods. Fifty male apolipoprotein E (ApoE) - - - mice were equally assigned to a normal group and a coronary AS group. In the AS group, the mice were given a high-fat diet to establish a coronary AS model. The macrophages of the mice were isolated for culture and divided into $7 \mathrm{groups}$ : normal, negative control (NC), control, miR-124-3p mimic, miR-124-3p inhibitor, si-MEKK3, and miR-124-3p inhibitor+si-MEKK3.

Results. Compared with the normal group, the AS group had lower expression levels of miR-124-3p and higher expression levels of MEKK3 and p-p38MAPK in the coronary artery tissue and peritoneal macrophages (all $p<0.050$ ). We found that miR-124-3p could negatively regulate MEKK3 expression. Compared with the control group, the miR-124-3p mimic group and si-MEKK3 group had greater cell apoptosis rates and Bax levels, weaker cell proliferation and invasion abilities, slower cell cycle progression, and lower PCNA and Bcl-2 levels (all $p<0.050$ ). This trend was also displayed in the miR-124-3p inhibitor+si-MEKK3 group when compared with the miR-124-3p inhibitor group, and in the si-MEKK3 group when compared with the miR-124-3p inhibitor+si-MEKK3 group (all $p<0.050$ ).
\end{abstract}

Conclusions. miR-124-3p overexpression can downregulate MEKK3 expression and inhibit the expression of the p38MAPK signaling pathway, thereby inhibiting macrophage proliferation and promoting macrophage apoptosis in mice with coronary AS.

Key words: MiR-124-3p, macrophage, coronary atherosclerosis 


\section{Introduction}

Atherosclerosis (AS) is the main cause of myocardial infarction and stroke. ${ }^{1}$ It is believed that both macrophages and monocytes play critical roles in AS. The occurrence of AS is associated with unstable plaque in which macrophages are among the main components. ${ }^{2}$ Recent studies have demonstrated that macrophages in the arterial plaque can proliferate, while differences exist in macrophage apoptosis between the early and late stages of $\mathrm{AS}^{3}$ Macrophage apoptosis in early stages can attenuate lesions, whereas apoptosis in the late stage is associated with AS plaque rupture. ${ }^{4,5}$

The mammalian genome contains hundreds of microRNAs (miRNAs), a type of non-coding RNA molecule of about 18-25 nucleotides regulating the expression of $30 \%$ of human genes. ${ }^{6}$ MiRNAs can inhibit the transcription and translation of target proteins, thereby affecting cell apoptosis, development and growth. ${ }^{7}$ Some studies have found that miRNA plays an essential part in the pathogenesis of AS through regulating multi-targets or signaling pathways. ${ }^{8,9}$ Recent studies have documented that homocysteine can downregulate the expression of miR-124-3p in AS, and its mechanism may be related to a change in hypermethylation in the promoter region of miR-124-3p. ${ }^{10}$ However, the role of miR-124-3p in AS is yet to be clarified.

As a mitogen-activated protein kinase (MAPK), MEKK3 has a vital role in cardiovascular development and can induce nuclear factor kappa-B activation. All eukaryotes have various MAPK pathways that control gene expression and cellular physiological activities. ${ }^{11}$ Normal MAPKs include extracellular signal-regulated kinase (ERK) $1 / 2$, JNK $1 / 2 / 3$, p38 $(\alpha, \beta, \gamma, \delta)$, and big MAPK (ERK5). ${ }^{12}$ So far, there has been little research on the role of MEKK3 in AS. The p38MAPK pathway, a member of the MAPK superfamily, is a key pathway in regulating cell proliferation and death, and can participate in many physiological processes. Meanwhile, the correlation between p38MAPK and MEKK3, another MAPK, cannot be neglected, as MEKK3 and MEKK4 are upstream regulators of p38 in many cells. ${ }^{13}$ Previous studies have documented that the MAPK pathway participates in cardiomegaly. ${ }^{14,15}$ However, the role of the MAPK pathway in the apoptosis and proliferation of macrophages in mice with coronary AS is unclear. The Targetscan bioinformatics website (www. targetscan.org) predicted that miR-124-3p can target MEKK3, and that miR-124-3p expression is downregulated in AS. Therefore, we infer that miR-124-3p may suppress the p38MAPK signaling pathway through downregulating MEKK3 expression, thereby inhibiting coronary AS and promoting the apoptosis of mouse macrophages.

In the present study, we established the AS model in apolipoprotein E (ApoE) knockout (ApoE -/-) mice and cultured mouse macrophages, which were then transfected with a miR-124-3p mimic, a miR-124-3p inhibitor,
si-MEKK3, and miR-124-3p inhibitor+si-MEKK3, respectively. RAW2647 cell apoptosis, cycle, proliferation, and invasion were examined in each group to investigate whether miR-124-3p can suppress phosphorylation of the p38MAPK signaling pathway by downregulating MEKK3 expression and finally affect the apoptosis and proliferation of macrophages in mice with AS.

\section{Material and methods}

\section{Animal subjects}

The study was approved by the Ethics Committee of Nankai University.

Fifty male ApoE -/- mice at 5 weeks of age were included as subjects and were assigned to a normal group and a coronary AS group (AS group), $n=25$ mice each. In the AS group, the mice were given a high-fat diet including $1 \%$ cholesterol and 15\% lard to establish a coronary AS mod$\mathrm{el}^{16}$; the mice in the normal group were given a normal diet. The feeding lasted 4 weeks and all the mice had access to water ad libitum. After 3 weeks, the coronary artery tissue from the AS and normal mice were collected for examination.

The mice were sacrificed and $3 \mathrm{~mL}$ of phosphate-buffered saline (PBS) was injected into the abdominal cavities. A gentle abdominal massage was applied to obtain the buffer from the abdomen. These steps were repeated 3 times. Afterward, the buffer was centrifuged $(300 \times \mathrm{g}$ for $15 \mathrm{~min})$, the supernatant was removed and the peritoneal macrophages in the precipitation were harvested. The cells were cultured in RPMI 1640 (Gibco, Thermo Fisher Scientific, Waltham, USA) which contained $10 \%$ fetal bovine serum (FBS), $50 \mathrm{U} / \mathrm{mL}$ of penicillin and $100 \mu \mathrm{g} / \mathrm{mL}$ of streptomycin (all from Gibco) at $37^{\circ} \mathrm{C}$ in an atmosphere of $5 \% \mathrm{CO}_{2}$ (incubator model: Thermo3111; Jinan Beisheng Medical Instruments Co. Ltd, Jinan, China). The medium was renewed after 2 days, and the cells were sub-cultured every 3-4 days. The cells in good condition and at the logarithmic phase were selected for transfection.

\section{Cell grouping and transfection}

The macrophages at the logarithmic phase from the AS mice were harvested and planted in a six-well culture plate (1*105/well). Serum-free RPMI 1640 medium without penicillin-streptomycin was used 1 day before transfection. The cells were assigned to 6 groups: the control group (macrophages from the AS mice), the negative control (NC) group (macrophages from the AS mice transfected with NC plasmid), the miR-124-3p mimic group (macrophage from the AS mice transfected with $4 \mu \mathrm{g}$ miR-124-3p mimic plasmid), the miR-124-3p inhibitor group (macrophages from the AS mice transfected with $4 \mu \mathrm{g}$ miR-124-3p inhibitor plasmid), the si-MEKK3 group (macrophages from 
$\mathrm{NC}$ :

miRNA-124 mimic:

miR-124 inhibitor: SiRNA:

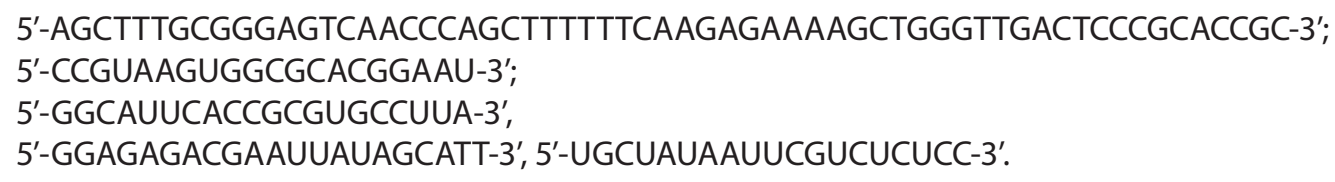

the AS mice transfected with $4 \mu \mathrm{g}$ of si-MEKK3 plasmid), and miR-124-3p inhibitor+si-MEKK3 group (macrophages from the AS mice co-transfected with $2 \mu \mathrm{g}$ miR-124-3p inhibitor plasmid and $2 \mu \mathrm{g}$ si-MEKK3 plasmid). The cells from the normal non-AS mice were cultured in a routine way without any other treatment.

The vectors used were constructed by Tianjin Saier Biotechnology Inc. (Tianjin, China) (see above).

Transfection was performed with Lipofectamine 2000 transfection reagent (Invitrogen, Carlsbad, USA) in accordance with the manufacturer's protocol. After $6 \mathrm{~h}$ of transfection, RPMI 1640 with 10\% FBS (Gibco) was used as the medium. The cells were harvested for subsequent experiments $48 \mathrm{~h}$ after transfection.

\section{Dual luciferase reporter assay}

The miR-124-3p binding site on MEKK3 was first analyzed on the www.targetscan.org bioinformatics website, followed by a dual luciferase reporter test to validate this relationship. MEKK3 dual luciferase reporter gene vectors with and without mutant miR-124-3p binding sites were constructed and named MEKK3mut and MEKK3wt, respectively. These 2 reporter plasmids were co-transfected with the NC mimic or miR-124-3p mimic into HEK $293 \mathrm{~T}$ cells. Renilla luciferase was used as a control. After $24 \mathrm{~h}$ of transfection, we performed a dual luciferase reporter assay. Cells from each group were lysed and then centrifuged $(4800 \times \mathrm{g}$ for $1 \mathrm{~min})$, and the supernatant was collected. A commercial dual luciferase reporter assay kit (Promega Biotech Co. Ltd., Beijing, China) was used in accordance with the manufacturer's instruction for measuring the activity of luciferase. The lysed cells were pipetted into Eppendorf tubes and $100 \mu \mathrm{L}$ of firefly luciferase solution was added to each $10 \mu \mathrm{L}$ sample. The activity of firefly luciferase was then measured, followed by the addition of $100 \mu \mathrm{L}$ of renilla luciferase solution to detect the activity of renilla luciferase. The formula is as follows: relative luciferase activity = firefly luciferase activity/renilla luciferase activity. ${ }^{8}$

\section{qRT-PCR}

After $48 \mathrm{~h}$ of transfection, cells were harvested from each group. Total RNAs were extracted using Trizol (16096020 from Thermo Fisher Scientific; and B1802 from HaiGene Bio, Quezon City, Philippines) followed by reverse transcription into cDNAs using TaqMan MicroRNA Assays Reverse Transcription Primer (Thermo Fisher Scientific). Next, SYBR ${ }^{\circledR}$ Premix ExTaq ${ }^{\mathrm{TM}}$ II kit (Xingzhi Biotech,
Table 1. qRT-PCR primer sequence

\begin{tabular}{|c|c|c|}
\hline Gene & Primer & Sequence \\
\hline \multirow{2}{*}{ miR-124-3p } & forward & 5'-GCGAGGATCTGTGAATGCCAAA-3' \\
\hline & reverse & 5'-AGGGGCTGCTTGAGTTGTAGTA-3' \\
\hline \multirow{2}{*}{ MEKK3 } & forward & 5'-TGTACCTGAGCGACAACAGC-3' \\
\hline & reverse & 5'-CACTGCTGAGGGGATCTAGC-3' \\
\hline \multirow{2}{*}{ p38MAPK } & forward & 5'-CCCGAACGATACCAGAACC-3' \\
\hline & reverse & 5'-GCGTGAATGATGGACTGAAA-3' \\
\hline \multirow{2}{*}{ PCNA } & forward & 5'-TCACGCCTGTGGTGGTTAC-3' \\
\hline & reverse & 5'-GGTCGGCTTCTGAGTTTCC-3' \\
\hline \multirow{2}{*}{$B C l-2$} & forward & 5'-AGTACCTGAACCGGCATCTG-3' \\
\hline & reverse & 5'-GCTGAGCAGGGTCTTCAGAG-3' \\
\hline \multirow{2}{*}{ Bax } & forward & 5'-CGAGCTGATCAGAACCATCA-3' \\
\hline & reverse & 5'-GGTCCCGAAGTAGGAGAGGA-3' \\
\hline \multirow{2}{*}{ U6 } & forward & 5'-GCTTCGGCAGCACATATACTAAAAT-3' \\
\hline & reverse & 5'-GCACTCCCGCCACAAAGATG-3' \\
\hline \multirow{2}{*}{ GAPDH } & forward & 5'-GGGAAATTCAACGGCACAGT-3' \\
\hline & reverse & 5'-AGATGGTGATGGGCTTCCC-3' \\
\hline
\end{tabular}

MAPK - mitogen-activated protein kinase; GAPDH - glyceraldehyde 3-phosphate dehydrogenase.

Guangzhou, China) was used for quantitative real-time polymerase chain reaction (qRT-PCR). Reagents were added in the following order: SYBR ${ }^{\circledR}$ Premix ExTaq ${ }^{\mathrm{TM}} \mathrm{II}$ $(2 \times, 25 \mu \mathrm{L})$, forward primer $(2 \mu \mathrm{L})$, reverse primer $(2 \mu \mathrm{L})$, ROX Reference Dye $(50 \times, 1 \mu \mathrm{L})$, DNA template $(4 \mu \mathrm{L})$, and double distilled $\mathrm{H}_{2} \mathrm{O}(16 \mu \mathrm{L})$. An ABI Prism ${ }^{\circledR} 7300$ fluorescence quantitative PCR instrument (Shanghai Kunke Instrument \& Equipment Co., Shanghai, China) was used to conduct qRT-PCR with the following running parameters: $95^{\circ} \mathrm{C}$ for $10 \mathrm{~min}$ (pre-denaturation), 32 cycles of $95^{\circ} \mathrm{C}$ for $15 \mathrm{~s}$ (denaturation) and $60^{\circ} \mathrm{C}$ for $30 \mathrm{~s}$ (annealing) before extension at $72^{\circ} \mathrm{C}$ for $1 \mathrm{~min} . \Delta \mathrm{Ct}=\mathrm{Ct}$ target gene $-\mathrm{Ct}_{\mathrm{GAPDH}}$; $\Delta \Delta \mathrm{Ct}=\Delta \mathrm{Ct}_{\text {study group }}-\Delta \mathrm{Ct}$ control group. U6 and GAPDH were the internal controls for $m i R-124-3 p$ and other genes, respectively. The relative gene expression of the target gene was calculated using the $2^{-\Delta \Delta \mathrm{Ct}}$ method. The primers are listed in Table 1.

\section{Western blot}

After $48 \mathrm{~h}$ of transfection and culture, the cells were washed 3 times in pre-cooled PBS. The total protein was extracted from the cells using RIPA lysis buffer (R0010; Solarbio Science \& Technology Co. Ltd., Beijing, China) containing phenylmethylsulfonyl fluoride (PMSF). The protein concentration was measured with a BCA kit (Thermo Fisher Scientific), and the deionized water was used for zeroing. 
The sample was then mixed with sample loading buffer and placed in a metal bath at $100^{\circ} \mathrm{C}$ for $10 \mathrm{~min}$. Afterward, a $50 \mu \mathrm{g}$ protein sample was loaded for $3 \mathrm{~h}$ of electrophoresis at $70 \mathrm{~V}$ constant voltage. The samples were then transferred to polyvinylidene difluoride (PVDF) membrane (ISEQ00010; Millipore Sigma, St. Louis, USA) using wet transfer at a constant current of $150 \mathrm{~mA}$. Subsequently, the membrane was blocked in skim milk (5\%) in TBST for $2 \mathrm{~h}$ at $4^{\circ} \mathrm{C}$ before the milk was discarded. The samples were washed in tris-buffered saline with Tween 20 (TBST) followed by the addition of polyclonal antibodies of rabbit anti-human MEKK3 (ab26321, 1:5000), anti-pp38MAPK (ab31828, 1:1000), anti-p38MAPK (ab31828, 1:5000), anti-PCNA (ab92552, 1:5000), anti-Bax (ab8805, 1:1000), anti-Bcl-2 (ab32124, 1:1000), and anti-GAPDH (ab8226, 1:2000, all from Abcam, Cambridge, UK) for incubation at $4^{\circ} \mathrm{C}$ overnight. Next, the samples were washed 3 times in TBST ( 6 min per wash) and treated for $2 \mathrm{~h}$ with horseradish peroxidase (HRP) labeled goat anti-rabbit IgG antibody (1:5000; Zhongshan Biotech, Beijing, China), followed by 3 more six-minute washes in TBST. Subsequently, the membrane was placed in TBS. Equal volumes of solutions A and B from an Excellent Chemiluminescent Substrate (ECL) kit (BB-3501; BestBio, Shanghai, China) were mixed, and $200 \mu \mathrm{L}$ was added to the membrane. Images were exposed in a gel-imager, photographed with an imaging analyzer (Bio-Rad Laboratories Inc, Hercules, USA), and analyzed using ImageJ software (National Institutes of Health, Bethesda, USA). The relative protein level = the grayscale value of the protein band/the grayscale value of the GAPDH protein band.

\section{MTT assay}

After $48 \mathrm{~h}$ of transfection and culture, the cells were digested routinely and harvested for counting. The cells were planted in a 96-well plate $\left(3-6^{*} 10^{3} /\right.$ well, $100 \mu \mathrm{L} /$ well), and each group had 6 wells. At 24 h, 48 h and 72 h, $20 \mu \mathrm{L}$ of $5 \mathrm{mg} / \mathrm{mL}$ MTT solution (Gibco) was added to each well. The plate was placed in the dark for $4 \mathrm{~h}$, and then $100 \mu \mathrm{L}$ of DMSO (Sigma-Aldrich, St. Louis, USA) was added into each well. The optical density (OD) value at $495 \mathrm{~nm}$ in each well was detected with a microplate reader (NYW-96M; Nuoyawei, Beijing, China). Cell viability curves were plotted with their OD value on the $y$-axis and time on the $x$-axis.

\section{Flow cytometry}

After $48 \mathrm{~h}$ of transfection, the cells were washed in PBS 3 times followed by centrifugation at $300 \times \mathrm{g}$ for $20 \mathrm{~min}$. Next, the supernatant was discarded and the cell concentration was controlled to $1 * 10^{5} / \mathrm{mL}$ using PBS. The sample was then treated with $1 \mathrm{~mL}$ of pre-cooled $75 \%$ ethanol (Shanghai Coking Co, Shanghai, China) at $4{ }^{\circ} \mathrm{C}$ for $1 \mathrm{~h}$, before being centrifuged at $250 \times \mathrm{g}$ for $5 \mathrm{~min}$ and washed 3 times in PBS. Afterward, $120 \mu \mathrm{L}$ of RNase A (Thermo Fisher
Scientific) was added in the dark, and the samples were water-bathed at $37^{\circ} \mathrm{C}$ for $40 \mathrm{~min}$ before being stained with $500 \mu \mathrm{L}$ of propidium iodide (PI; Sigma-Aldrich) in the dark at $4^{\circ} \mathrm{C}$ for $30 \mathrm{~min}$. The cell cycle was examined with a flow cytometer (Beckman Coulter Inc., Brea, USA) at $488 \mathrm{~nm}$.

After $48 \mathrm{~h}$ of transfection, the cells were digested with EDTA-free pancreatin (Thermo Fisher Scientific) and placed in flow cytometry tubes for centrifugation $(300 \times \mathrm{g}$ for $30 \mathrm{~min})$. Next, the supernatant was discarded, and the cells were washed 3 times in pre-cooled PBS before centrifugation $(300 \times \mathrm{g}$ for $20 \mathrm{~min})$. The supernatant was discarded, and annexin V-fluorescein isothiocyanate (FITC)/PI dye was prepared using HEPES buffer (Thermo Fisher Scientific) annexin V-FITC and PI (50:1:2) according to the instructions in a commercial Annexin-V-FITC Cell Apoptosis Detection Kit (Sigma-Aldrich). Subsequently, the samples were mixed with $100 \mu \mathrm{L}$ of dye and incubated at room temperature for 15 min before being mixed with HEPES buffer (Thermo Fisher Scientific). A flow cytometer was used to detect cell apoptosis. The green fluorescence of annexin V-FITC was examined through the FITC channel (FL1), while the red fluorescence of PI was examined through the PI channel (FL2 or FL3). The excitation and emission wavelengths were $488 \mathrm{~nm}$ and $530 \mathrm{~nm}$, respectively.

\section{Cell invasion}

Transwell chambers (Jrdun Biotechnology Co., Shanghai, China) were put in a 96-well plate. The upper chambers were coated with Matrigel (1:8, Sigma-Aldrich) and then dried at room temperature. The cells were digested with pancreatin (Sigma-Aldrich) and washed 3 times in PBS. The cells were then resuspended in RPMI 1640 medium, and the density was adjusted to $1 * 10^{5} / \mathrm{mL}$. Cell suspension $(300 \mu \mathrm{L})$ was added to the Matrigel-coated upper chambers, and $500 \mu \mathrm{L}$ of RPMI 1640 medium with $10 \%$ FBS (Gibco) was added to the lower chambers. After culturing for $24 \mathrm{~h}$, the transwell chambers were taken, and the non-invaded cells in the upper chamber were gently scraped off using cotton swabs. Subsequently, the samples were fixed with paraformaldehyde (4\%) (Beijing Leagene Biotechnology Co. Ltd., Beijing, China) for $20 \mathrm{~min}$, stained with crystal violet ( $0.5 \%$ Solarbio) and then washed 3 times in PBS. Under an inverted microscope, 5 fields were randomly selected for photographing ( $\times 200$ magnification), and the cells passing through the membrane were counted.

\section{Statistical analysis}

The SPSS v. 21.0 software (IBM Corp., Armonk, USA) was used to analyze the data. Measurement data are expressed as means \pm standard deviation (SD). One-way analysis of variance (ANOVA) was used for multiple group comparisons, and Tukey's post hoc test for pairwise comparisons was used between multiple groups. A value of $\mathrm{p}<0.050$ indicated a statistically significant difference. 


\section{Results}

\section{Expressions of miR-124-3p, MEKK3 and $p$-p38MAPK in the coronary arteries of mice}

To investigate the expressions of miR-124-3p and MEKK3 and the activation of the p38MAPK signaling pathway in the AS and the normal groups, we measured the expression levels of miR-124-3p and MEKK3 using qRT-PCR, and the phosphorylation of p38MAPK, a factor related to the p38MAPK signaling pathway, using western blot. Compared with the normal group, the miR-124-3p expression level was lower in the coronary arteries in the AS group ( $\mathrm{p}=0.001$ ), and the phosphorylation levels of MEKK3 and $\mathrm{p} 38 \mathrm{MAPK}$ were higher in the AS group ( $\mathrm{p}=0.001$ and $\mathrm{p}=0.0006$, respectively). Meanwhile, the basal expression level of the p38MAPK protein was similar in the 2 groups $(\mathrm{p}=0.340)$ (Fig. 1).

\section{Mir-124-3p can negatively regulate the MEKK3 gene}

The bioinformatics website www.microrna.org/microrna/home.do predicted the existence of a miR-124-3p binding site on MEKK3 (Fig. 2A). Figure 2B shows that compared with the groups transfected with the $\mathrm{NC}$ mimic, luciferase activity was much lower in the group
A

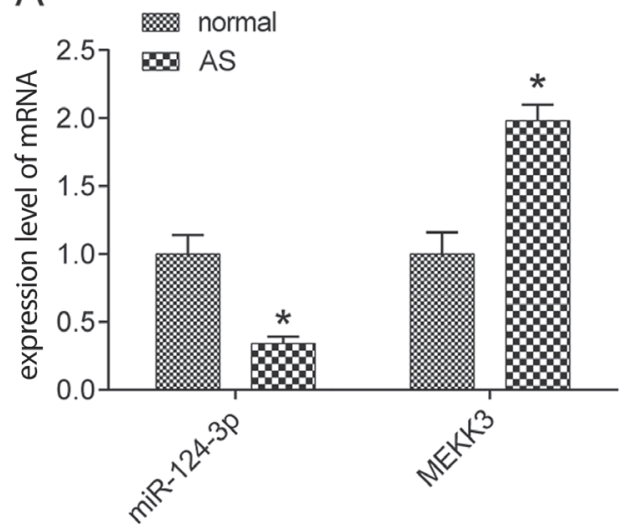

B

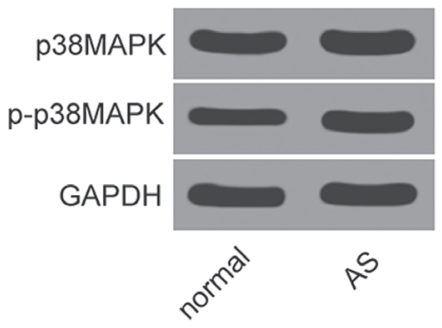

C

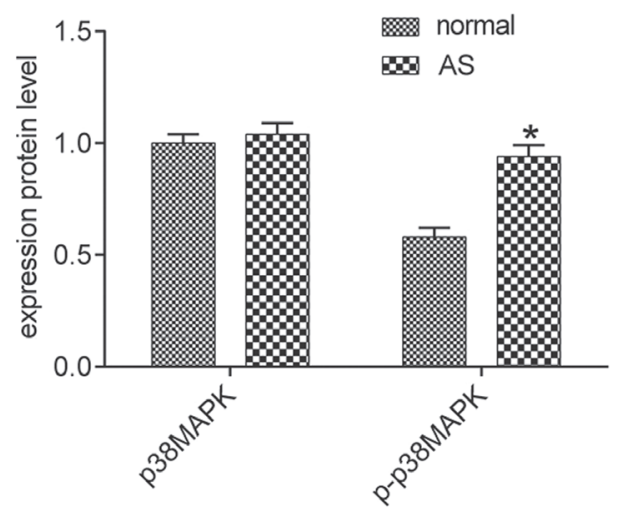

Fig. 1. Expressions of miR-124-3p, MEKK3, p38MAPK, and p-p38MAPK in the normal and AS groups. A. Histogram of miR-124-3p and MEKK3 mRNA expression levels in the tissues. B. Protein bands of p38MAPK and p-p38MAPK in the tissues. C. Histogram of protein levels of p38MAPK and p-p38MAPK in the tissues

${ }^{*} \mathrm{p}<0.050$ vs the normal group; MAPK - mitogen-activated protein kinase; GAPDH - glyceraldehyde 3-phosphate dehydrogenase

A

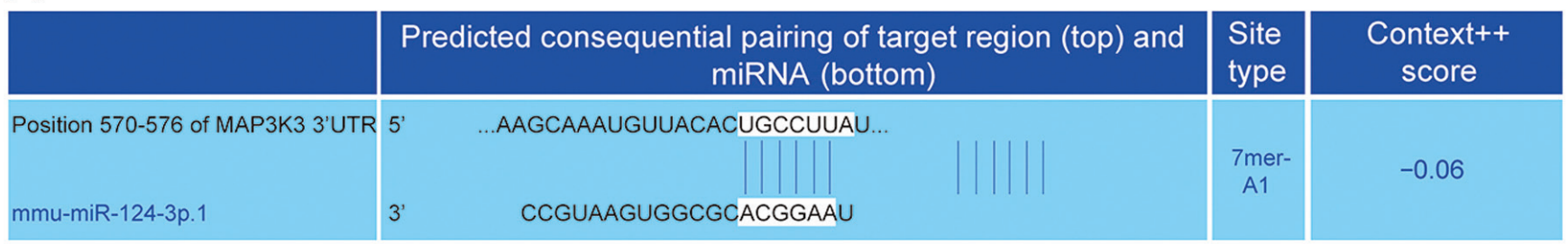
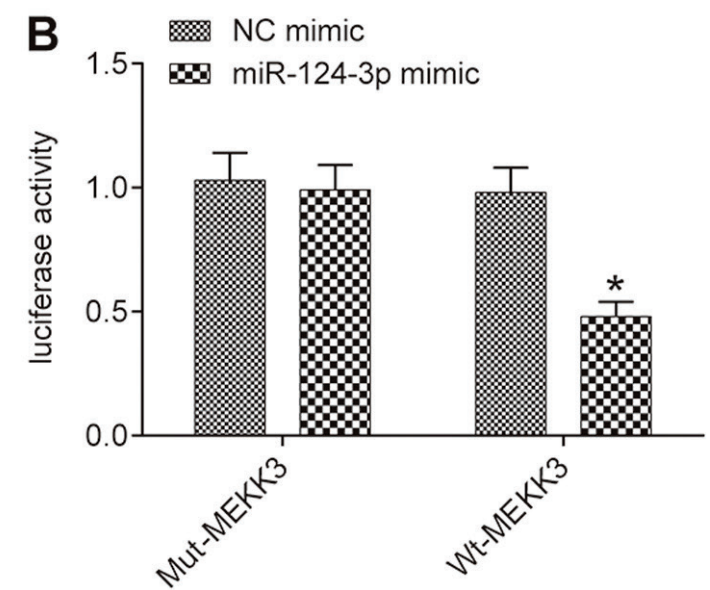

Fig. 2. MiR-124-3p can negatively regulate the MEKK3 gene. A. 3'-UTR sequence for the paring of miR-124-3p and MEKK3. B. Luciferase activity 

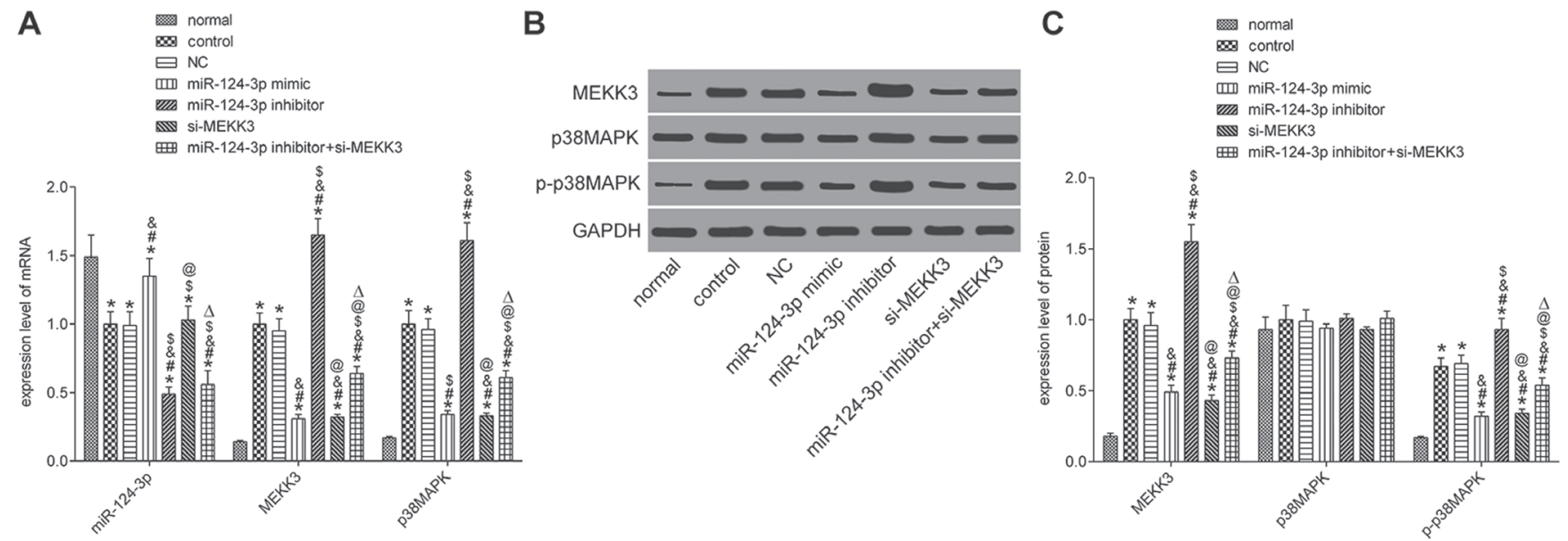

Fig. 3. MiR-124-3p, MEKK3 and p38MAPK expressions in each group. A. Histogram of miR-124-3p, MEKK3 and p38MAPK mRNA levels. B. Protein bands of MEKK3, p38MAPK and p-p38MAPK. C. Histogram of MEKK3, p38MAPK and p-p38MAPK protein levels

${ }^{*} p<0.050$ vs the normal group; ${ }^{p} p<0.050$ vs the control group; ${ }^{\circledR} p<0.050$ vs the NC group; ${ }^{\$} p<0.050$ vs the mR-124-3p mimic group; ${ }^{@} p<0.050$ vs the miR-124-3p inhibitor group; ${ }^{\Delta} p<0.050$ vs the si-MEKK3 group; MAPK - mitogen-activated protein kinase; GAPDH - glyceraldehyde 3-phosphate dehydrogenase.

co-transfected with Wt-MEKK3 and miR-124-3p mimic $(p=0.001)$. At the same time, luciferase activity remained unchanged in the group co-transfected with MEKK3mut $(\mathrm{p}=0.665)$. This finding indicates that miR-124-3p can negatively regulate the $M E K K 3$ gene.

\section{Mir-124-3p, MEKK3 and p38MAPK expressions in each group}

In order to investigate the mechanism of how miR1243p mediates the $\mathrm{p} 38 \mathrm{MAPK}$ signaling pathway through the $M E K K 3$ gene and exerts effects on macrophage apoptosis and proliferation in AS mice, we measured the miR-124-3p expression level and mRNA expression levels of MEKK3 and p38MAPK using qRT-PCR, and measured MEKK3 protein expression and the phosphorylation of P38MAPK using western blot. As shown in Fig. 3, compared with the normal group, the other groups had lower levels of miR-124-3p and higher phosphorylation levels of MEKK3 and p38MAPK (all p < 0.0001); meanwhile, the basal protein expression level of p38MAPK was similar in the normal group and the other groups ( $p=0.449)$. Compared with the control group, the NC group had similar expression levels of each gene; whereas the expression levels of MEKK3 and p-p38MAPK were lower in the miR-124-3p mimic, si-MEKK3 and miR-124-3p inhibitor+si-MEKK3 groups (all p < 0.0001); and the expression levels of MEKK3 and p-p38MAPK were higher in the miR-124-3p inhibitor group ( $\mathrm{p}=0.001, \mathrm{p}=0.003)$. The miR-124-3p inhibitor $+\mathrm{si}-$ MEKK3 group had higher expression levels of MEKK3 and p-p38MAPK than the si-MEKK3 group ( $\mathrm{p}=0.0005$, $\mathrm{p}=0.0008)$. Compared with the miR-124-3p inhibitor group, the miR-124-3p inhibitor+si-MEKK3 group had lower expression levels of MEKK3 and p-p38MAPK (both $\mathrm{p}=0.0002$ ).

\section{Cell proliferation}

The MTT assay results showed that cell growth increased with time. The normal group had lower OD values at $24 \mathrm{~h}$ and $48 \mathrm{~h}$ than the other groups (all $\mathrm{p}<0.0001$ ). In comparison with the control group, the $\mathrm{NC}$ group had similar OD values at $24 \mathrm{~h}$ and $48 \mathrm{~h}(\mathrm{p}=0818, \mathrm{p}=0.744)$, while the OD values at $48 \mathrm{~h}$ and $72 \mathrm{~h}$ were lower in the miR-124-3p mimic group, the si-MEKK3 group and the miR124 -inhbitor+si-MEKK3 groups $(\mathrm{p}=0.005, \mathrm{p}=0.009)$, and higher in the miR-124-3p inhibitor group ( $\mathrm{p}=0.018$, $\mathrm{p}=0.032$ ). Meanwhile, the miR-124-3p inhibitor+si-MEKK3 group had higher OD values at $48 \mathrm{~h}$ and $72 \mathrm{~h}$ than the si-MEKK3 group ( $\mathrm{p}=0.030, \mathrm{p}=0.039)$, and lower OD values at $48 \mathrm{~h}$ and $72 \mathrm{~h}$ than the miR-124-3p inhibitor group $(\mathrm{p}=0.030, \mathrm{p}=0.039)$ (Fig. 4).

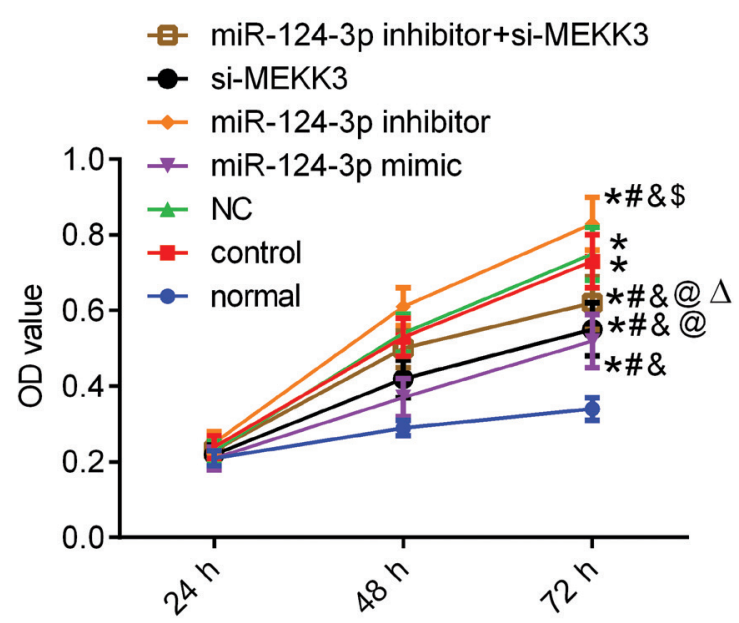

Fig. 4. Cell proliferation measured using MTT assay

${ }^{*} p<0.050$ vs the normal group; ${ }^{*} p<0.050$ vs the control group; ${ }^{\&} p<0.050$ vs the NC group; ${ }^{\$} p<0.050$ vs the mR-124-3p mimic group; ${ }^{@} \mathrm{p}<0.050$ vs the miR-124-3p inhibitor group; ${ }^{\Delta} p<0.050$ vs the si-MEKK3 group; OD - optical density. 


\section{Cell cycle}

The flow cytometry results displayed that compared to the normal group, the other groups had fewer $\mathrm{G} 1$ phase cells and more $S$ phase cells (both $\mathrm{p}<0.0001$ ). There were no differences between the control and $\mathrm{NC}$ groups in terms of the cell ratios at the $\mathrm{G} 1, \mathrm{~S}$ and $\mathrm{G} 2$ phases $(\mathrm{p}=0.982, \mathrm{p}=0.802, \mathrm{p}=0.756)$. Compared with the control group, the miR-124-3p mimic, si-MEKK3 and miR-124-3p inhibitor+si-MEKK3 groups had more G1 phase cells and fewer S phase cells $(\mathrm{p}=0.0002$, $\mathrm{p}<0.0001$ ), whereas the miR-124-3p inhibitor group had fewer G1 phase cells and more $S$ phase cells $(\mathrm{p}=0.003, \mathrm{p}=0.008)$. Meanwhile, the miR-124-3p inhibitor+si-MEKK3 group had fewer $\mathrm{G} 1$ cells and more $\mathrm{S}$ cells than the si-MEKK3 group ( $p=0.020, p=0.002)$, and more G1 phase cells and fewer $\mathrm{S}$ phase cells than the miR-124-3p inhibitor group $(\mathrm{p}=0.001$, $\mathrm{p}=0.0004$ ) (Fig. 5).

\section{Cell apoptosis}

The flow cytometry results showed that compared to the normal group, the other groups had lower cell apoptosis rates $(p<0.0001)$. There was no difference between the control and $\mathrm{NC}$ groups in terms of the cell apoptosis rate $(\mathrm{p}=0.528)$. Compared with the control group, the miR-124-3p mimic, si-MEKK3 and miR-124-3p inhibitor+si-MEKK3 groups had higher cell apoptosis rates $(\mathrm{p}<0.0001)$, and the miR-124-3p inhibitor group had a lower cell apoptosis rate $(\mathrm{p}=0.0002)$. The miR-124-3p inhibitor+si-MEKK3 group had a lower cell apoptosis rate than the si-MEKK3 group and a higher apoptosis rate than the miR-124-3p inhibitor group (both $\mathrm{p}<0.0001$ ) (Fig. 6).
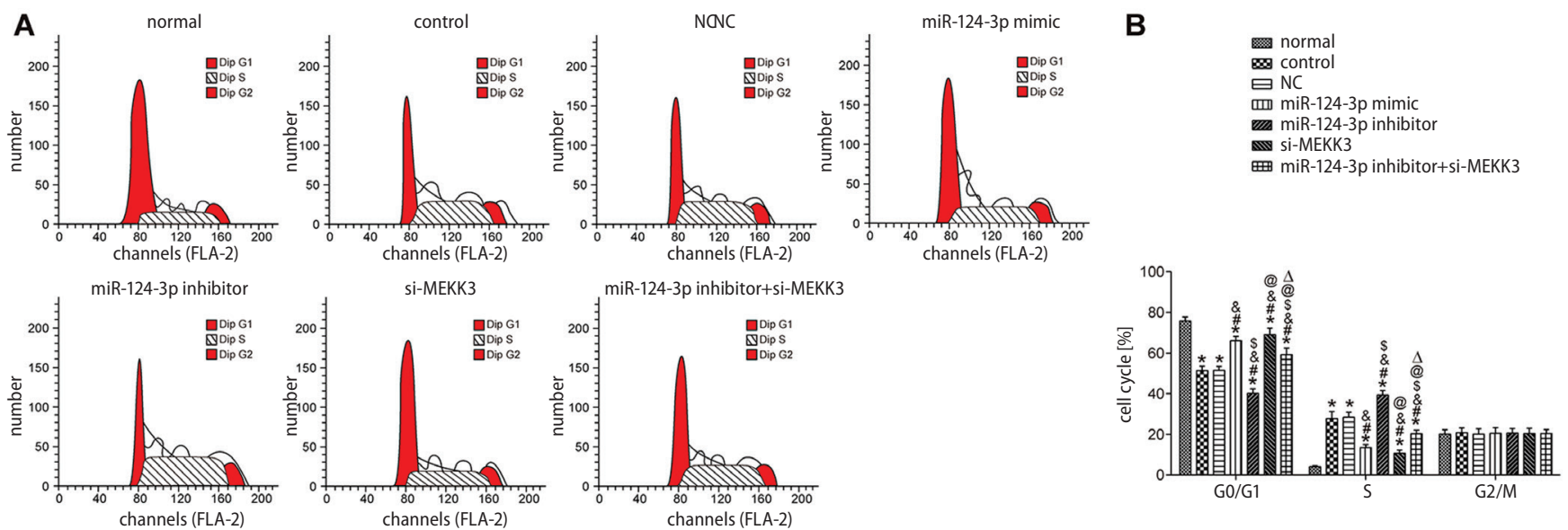

Fig. 5. Cell cycle detected with flow cytometry. A. Cell cycle in each group. B. Histogram of a cell cycle

${ }^{*} p<0.050$ vs the normal group; ${ }^{p} p<0.050$ vs the control group; ${ }^{\circledR} p<0.050$ vs the NC group; ${ }^{\$} p<0.050$ vs the miR-124-3p mimic group; ${ }^{@} p<0.050$ vs the miR-124-3p inhibitor group; ${ }^{\wedge} p<0.050$ vs the si-MEKK3 group.

A
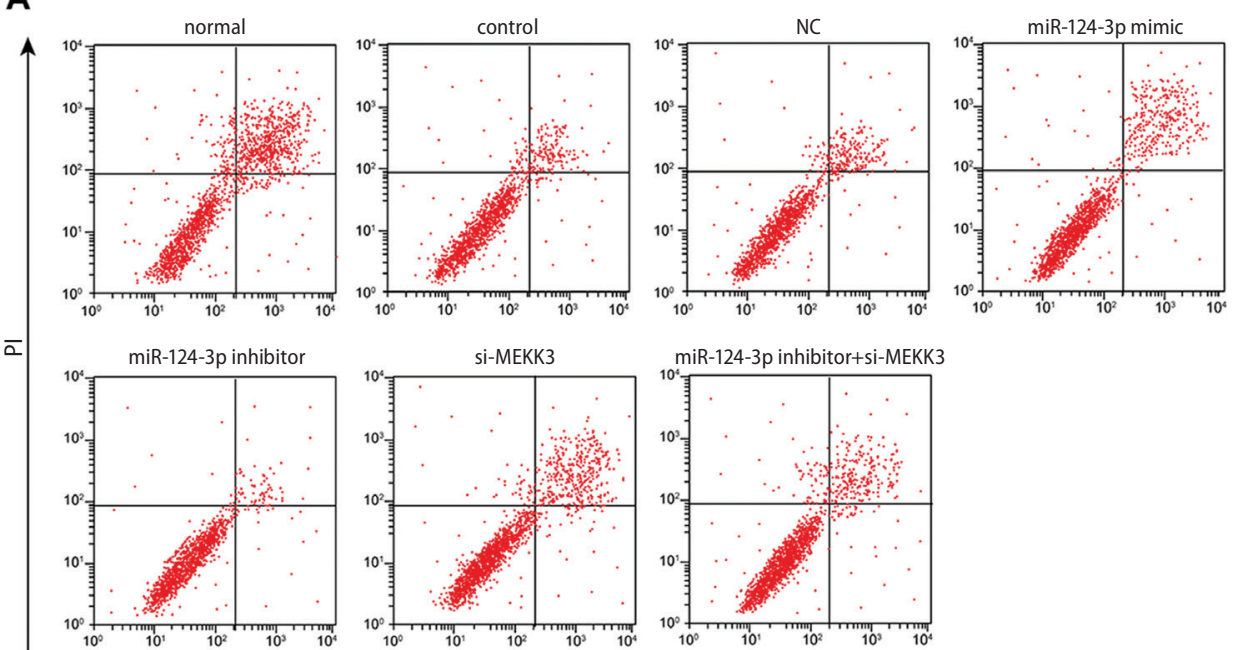

miR-124-3p inhibitor+si-MEKK3

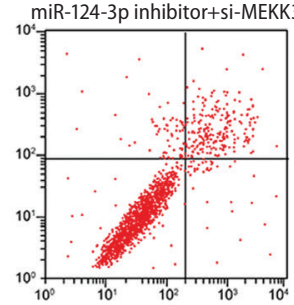

B

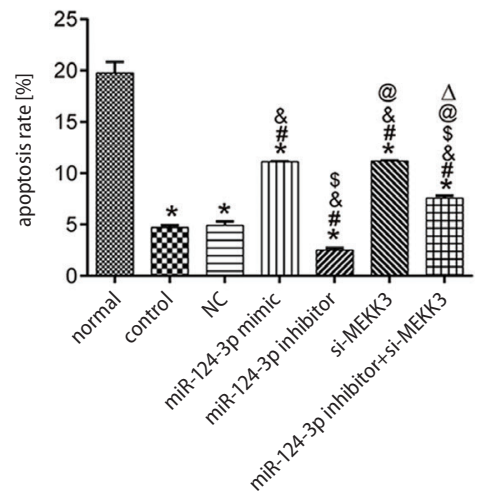

Fig. 6. Cell apoptosis detected using flow cytometry. A. Cell apoptosis rate. B. Histogram of the cell apoptosis rate

${ }^{*} p<0.050$ vs the normal group; ${ }^{p}<0.050$ vs the control group; ${ }^{*} p<0.050$ vs the NC group; ${ }^{\$} p<0.050$ vs the mR-124-3p mimic group; ${ }^{\circledR} p<0.050$ vs the miR-124-3p inhibitor group; ${ }^{\Delta} p<0.050$ vs the si-MEKK3 group; FITC - fluorescein isothiocyanate. 


\section{Cell invasion}

The transwell assay results showed that compared to the normal group, the other groups had higher numbers of invading cells ( $\mathrm{p}<0.0001)$. No difference was observed in the number of invading cells between the control and NC groups $(\mathrm{p}=0.276)$. Compared with the control group, the miR-124-3p mimic, si-MEKK3 and miR-124-3p inhibitor+si-MEKK3 groups had fewer invading cells ( $\mathrm{p}<0.0001)$, and the miR-124-3p inhibitor group had more invading cells $(\mathrm{p}=0.039)$. Moreover, the miR-124-3p inhibitor+si-MEKK3 group had a higher cell apoptosis rate than the si-MEKK3 group ( $p=0.007)$ and fewer invading cells than the miR-124-3p inhibitor group $(p=0.003)$ (Fig. 7).

\section{mRNA and protein expression levels of PCNA, BCl-2 and Bax}

To investigate how miR-124-3p affects the apoptosis and proliferation of macrophages in mice with AS by mediating the p38MAPK signaling pathway through the $M E K K 3$ gene, we measured the mRNA and protein expression levels of proliferation-associated factor PCNA and apoptosisassociated factors Bcl-2 and Bax using qRT-PCR and western blot. The results showed that compared to the normal group, the other groups had higher mRNA and protein expression levels of PCNA and $\mathrm{Bcl}-2$, and lower mRNA and protein expression levels of $\mathrm{Bax}$ (all $\mathrm{p}<0.0001)$. The expression levels of each gene in the control and NC groups were similar. Compared with the control group, the miR-124-3p mimic, si-MEKK3, miR-124-3p inhibitor+si-MEKK3 groups had lower PCNA and Bcl-2 mRNA and protein expression levels (PCNA mRNA, $\mathrm{p}=0.0004, \mathrm{Bcl}-2$
mRNA $\mathrm{p}<0.0001$, PCNA protein $\mathrm{p}<0.0001$, Bcl-2 protein $\mathrm{p}<0.0001$ ), and higher Bax mRNA and protein expression levels $(\mathrm{p}=0.0002, \mathrm{p}<0.0001)$, while the miR-124-3p inhibitor group had greater PCNA and Bcl-2 mRNA and protein expression levels (PCNA mRNA p $=0.0005, \mathrm{Bcl}-2$ mRNA $\mathrm{p}=0.004$, PCNA protein $\mathrm{p}=0.001, \mathrm{Bcl}-2$ protein $\mathrm{p}=0.001$ ), and lower Bax mRNA and protein expression levels $(p=0.010, p=0.002)$. The miR-124-3p inhibitor+siMEKK3 group had higher PCNA and Bcl-2 mRNA and protein expression levels (PCNA mRNA $\mathrm{p}=0.035$, Bcl-2 mRNA $\mathrm{p}=0.004$, PCNA protein $\mathrm{p}=0.0005, \mathrm{Bcl}-2$ protein $\mathrm{p}=0.003)$, and lower Bax mRNA and protein expression levels ( $\mathrm{p}=0.011, \mathrm{p}<0.0001$ ) than the si-MEKK3 group, and lower PCNA and $\mathrm{Bcl}-2 \mathrm{mRNA}$ and protein expression levels (PCNA mRNA $\mathrm{p}=0.0002, \mathrm{Bcl}-2$ mRNA $\mathrm{p}=0.001$, PCNA protein $\mathrm{p}<0.0001, \mathrm{Bcl}-2$ protein $\mathrm{p}<0.0001)$, and higher Bax mRNA and protein expression levels $(\mathrm{p}=0.0008$, $\mathrm{p}=0.003$ ) than the miR-124-3p inhibitor group (Fig. 8).

\section{Discussion}

Macrophage apoptosis takes part in the whole process of AS and serves a critical role in the pathogenesis of this disease. Macrophage cells are a major component of AS plaque. The plaque is likely to be ruptured if it is unstable, and once the plaque is ruptured, a thrombus can easily form. ${ }^{17}$ Macrophages play an essential part in all stages of AS, including lesion occurrence and expansion, necrosis that leads to rupture, the clinical manifestation of AS, and even the subsidence of AS. ${ }^{18,19}$ Different phenotypes can be exhibited in macrophages based on the environment and the activation of intracellular signaling pathways. As researchers are gaining more understanding about the role
A
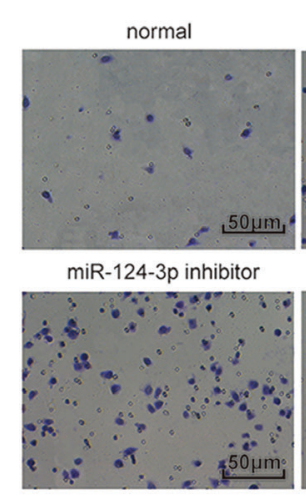

control

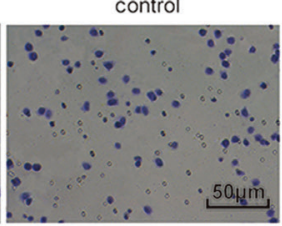

Si-MEKK3

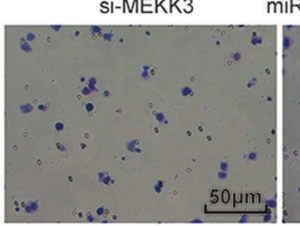

NC

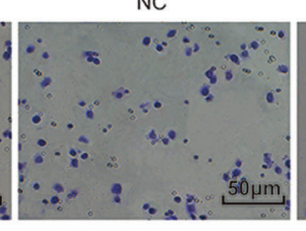

miR-124-3p inhibitor+si-MEKK3

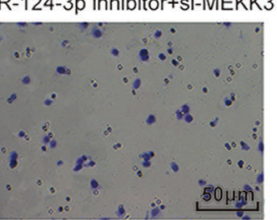

B

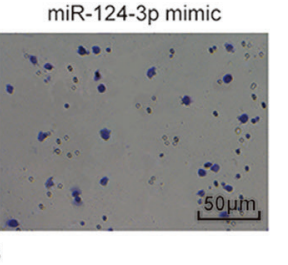

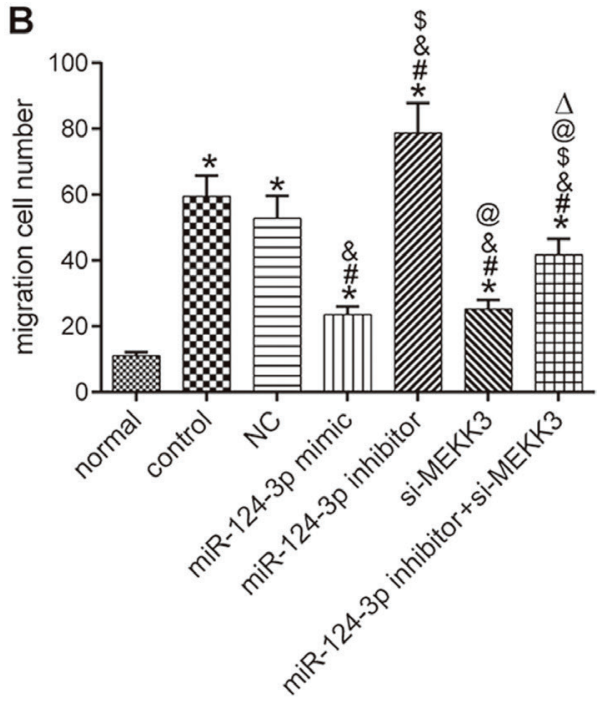

Fig. 7. Cell invasion measured using transwell assay. A. Image of cell invasion ( $\times 200$ magnification). B. Histogram of the number of invading cells in each group

${ }^{*} p<0.050$ vs the normal group; ${ }^{*} p<0.050$ vs the control group; ${ }^{\&} p<0.050$ vs the NC group; $\$ p<0.050$ vs the mR-124-3p mimic group; ${ }^{@} p<0.050$ vs the miR-124-3p inhibitor group; ${ }^{\Delta} p<0.050$ vs the si-MEKK3 group. 

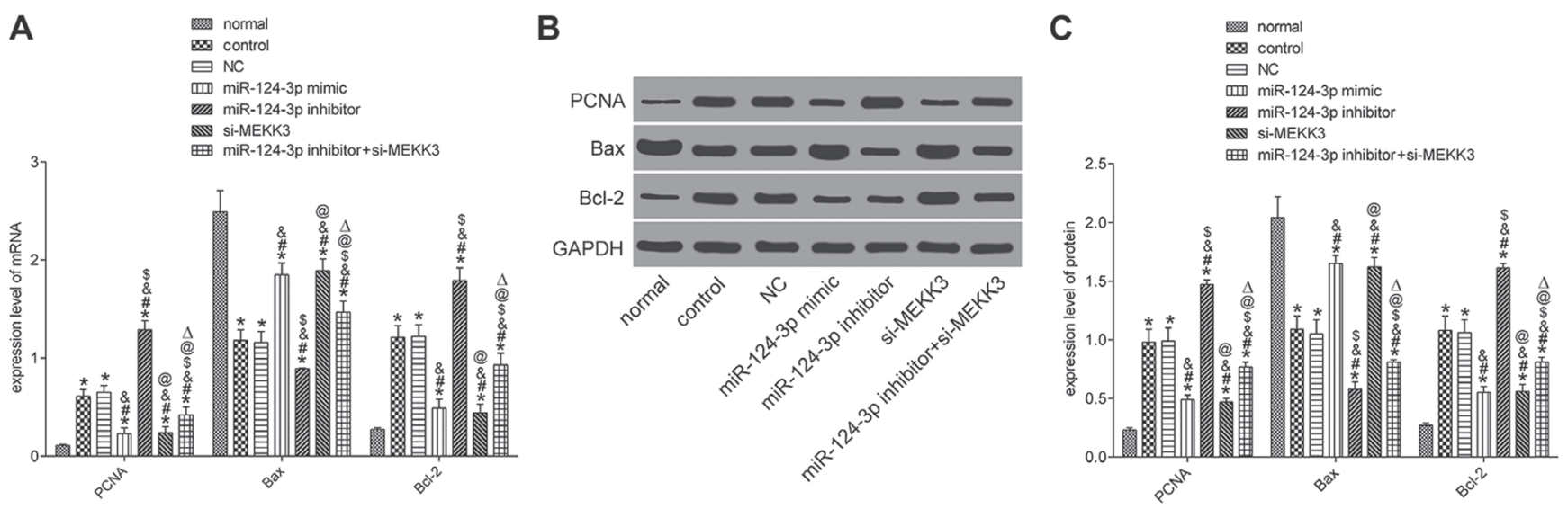

Fig. 8. RNA and protein expression levels of PCNA, Bax and BCl-2. A. Histogram of PCNA, Bax and BCl-2 mRNA levels. B. Protein bands of PCNA, Bax and BCl-2. C. Histogram of protein levels of PCNA, Bax and BCl-2 in each group

${ }^{*} p<0.050$ vs the normal group; ${ }^{p} p<0.050$ vs the control group; ${ }^{\&} p<0.050$ vs the NC group; $\$ p<0.050$ vs the miR-124-3p mimic group; ${ }^{\circledR} p<0.050$ vs the miR-124-3p inhibitor group; ${ }^{\Delta} p<0.050$ vs the si-MEKK3 group.

of inflammatory macrophages, molecular therapy is now emerging. ${ }^{19,20}$

In recent years, miR-124-3p downregulation has been observed in various types of human diseases, including Parkinson's disease, AS, and nerve damage. ${ }^{21-23}$ Activation of the MAPK pathway is a major intracellular event in cellular responses to environmental stress. Some studies have revealed that MEKK3, a MAP3K family member, can be strongly expressed in human and mice platelets, and the lack of platelet-specific MEKK3 can hinder arterial thrombus formation; moreover, MEKK3 can activate ERK1/2 and JNK2 in platelets and cause microthrombus. ${ }^{24}$ p38MAPK signaling serves a critical role in regulating cell apoptosis and proliferation. Some studies have demonstrated that suppressing p38MAPK phosphorylation in cardiomyocyte can reduce oxidative injury, whereas p38MAPK phosphorylation in bladder cancer cells can promote the occurrence of tumors. ${ }^{25,26}$ Meanwhile, it has also been reported that $\mathrm{p} 38 \mathrm{MAPK}$ phosphorylation can aggravate inflammation in fibroblasts and increase inflammatory activity in the retina. ${ }^{27,28}$ However, the effect of the p38MAPK signaling pathway in AS remains unclear.

Studies have indicated that the expression level of MEKK3 is elevated in thrombi and that high MEKK3 expression can promote phosphorylation of the p38MAPK signaling pathway via activation of the MAPK pathway, thereby affecting chondriosome and cell nuclei. ${ }^{29}$ In the present study, we found that miR-124-3p expression was downregulated in macrophages and in the tissues of AS mice, while MEKK3 and the phosphorylation of $\mathrm{p} 38 \mathrm{MAPK}$ signaling pathway were upregulated. In this study, the MEKK3 interference sequence was transfected into the macrophages, and this interference caused marked decreases in p38MAPK phosphorylation, expressions of the proliferation-associated factor PCNA and the antiproliferative factor Bcl-2, cell proliferation and invasion abilities, cell cycle progression, and the interference increased the expression of the pro-apoptotic factor Bax and the cell apoptosis rate. The results indicated that MEKK3 interference can suppress the phosphorylation of p38MAPK signaling pathway, thereby inhibiting macrophage proliferation and invasion, and promoting macrophage apoptosis in mice, which aligned with previous studies. ${ }^{13,29}$

To further investigate MEKK3 upstream regulation, we checked the www.microrna.org/microrna/home.do bioinformatics website and found that miR-124-3p can target MEKK3. It has also been documented that miR-124-3p has inhibitory effects on various diseases. ${ }^{30}$ Our dual luciferase reporter assay suggested that miR-124-3p can negatively regulate MEKK3. We performed transfections of miR-124-3p mimic, miR-124-3p inhibitor and miR-124-3p inhibitor+si-MEKK3 into mice macrophages and found that miR-124-3p overexpression can inhibit the proliferation and invasion of macrophages and promote their apoptosis, whereas miR-124-3p silencing can achieve the opposite effect. Compared with the miR-124-3p inhibitor group, macrophages in the miR-124-3p inhibitor+siMEKK3 group had weaker proliferation and invasion abilities, higher apoptosis rates and lower p38MAPK phosphorylation, suggesting that inhibiting MEKK3 expression can partially counteract the effect of miR-124-3p silencing on macrophage growth, thereby inhibiting the phosphorylation of the p38MAPK signaling pathway. These results demonstrated that miR-124-3p overexpression can inhibit MEKK3 gene expression and the phosphorylation of the $\mathrm{p} 38 \mathrm{MAPK}$ signaling pathway in the AS mouse model, thereby inhibiting macrophage proliferation and invasion, and promoting microphage apoptosis.

\section{Conclusions}

MiR-124-3p can mediate the p38MAPK signaling pathway by targeting the $M E K K 3$ gene, thereby inhibiting macrophage growth. The pathogenesis of AS was further 
elucidated in this study, which can contribute to the theoretical basis for treating AS. However, more clinical data needs to be included, and the way in which how MEKK3 participates in the p38MAPK signaling pathway needs to be investigated in future studies in order to verify our results and further clarify the relationship between miR-124-3p and AS, the inhibitory effect of miR-124-3p on MEKK3 and the effect of miR-124-3p in AS.

\section{ORCID iDs}

Chuannan Zhai (1) https://orcid.org/0000-0003-3504-5957 Hongliang Cong (1) https://orcid.org/0000-0001-6525-064X Kai Hou (1) https://orcid.org/0000-0002-0341-8117

Yuecheng Hu (1) https://orcid.org/0000-0001-7292-3996

Jingxia Zhang (1) https://orcid.org/0000-0002-4936-7554

Yingyi Zhang (10) https://orcid.org/0000-0002-9134-7055

Ying Zhang (1) https://orcid.org/0000-0003-2639-504X

Hong Zhang (D) https://orcid.org/0000-0002-0650-0482

\section{References}

1. Babaev VR, Huang J, Ding L, Zhang Y, May JM, Linton MF. Loss of rictor in monocyte/macrophages suppresses their proliferation and viability reducing atherosclerosis in LDLR null mice. Front Immunol. 2018;9:215.

2. Kruth HS. Fluid-phase pinocytosis of LDL by macrophages: A novel target to reduce macrophage cholesterol accumulation in atherosclerotic lesions. Curr Pharm Des. 2013;19(33):5865-5872.

3. McCurdy S, Baumer Y, Toulmin E, Lee BH, Boisvert WA. Macrophagespecific expression of IL-37 in hyperlipidemic mice attenuates atherosclerosis. J Immunol. 2017;199(10):3604-3613.

4. Xiao $Y$, He M, Liang $X$, et al. Pu-erh tea ameliorates atherosclerosis associated with promoting macrophage apoptosis by reducing NF-kappaB activation in ApoE knockout mice. Oxid Med Cell Longev. 2018;2018:3197829.

5. Gonzalez L, Qian AS, Tahir U, Yu P, Trigatti BL. Sphingosine-1-phosphate receptor 1, expressed in myeloid cells, slows diet-induced atherosclerosis and protects against macrophage apoptosis in Ldlr KO mice. Int J Mol Sci. 2017;18(12):2721.

6. Zhang YL, Li XB, Hou YX, Fang NZ, You JC, Zhou QH. The IncRNA XIST exhibits oncogenic properties via regulation of miR-449a and $\mathrm{BCl}-2$ in human non-small cell lung cancer. Acta Pharmacol Sin. 2017;38(3): 371-381 [Erratum in: The IncRNA XIST exhibits oncogenic properties via regulation of miR-449a and $\mathrm{Bcl}-2$ in human non-small cell lung cancer. Acta Pharmacol Sin. 2017;38(3):443.

7. O'Connell RM, Rao DS, Chaudhuri AA, Baltimore D. Physiological and pathological roles for microRNAs in the immune system. Nat Rev Immunol. 2010;10(2):111-122.

8. Song K, Li L, Sun G, Wei Y. MicroRNA-381 regulates the occurrence and immune responses of coronary atherosclerosis via cyclooxygenase-2. Exp Ther Med. 2018;15(5):4557-4563.

9. Ahmadi R, Heidarian E, Fadaei R, Moradi N, Malek M, Fallah S. miR-342-5p expression levels in coronary artery disease patients and its association with inflammatory cytokines. Clin Lab. 2018;64(4):603-609.

10. Wang $X$, Lian $Y$, Wen $X$, et al. Expression of miR-126 and its potential function in coronary artery disease. Afr Health Sci. 2017;17(2):474-480.

11. Zhao $L$, Jiao $Y$, Yang AN, et al. The effect of miR-124 on homocysteine-induced atherosclerosis via promoter region DNA methylation in ApoE(-/-) mice [in Chinese]. Sheng LiXue Bao. 2015;67(2):207-213.
12. Yang J, Lin Y, Guo Z, et al. The essential role of MEKK3 in TNF-induced NF-kappaB activation. Nat Immunol. 2001;2(7):620-624.

13. Padda R, Wamsley-Davis A, Gustin MC, Ross R, Yu C, Sheikh-Hamad D. MEKK3-mediated signaling to p38 kinase and TonE in hypertonically stressed kidney cells. Am J Physiol Renal Physiol. 2006;291(4): F874-F881.

14. Feng $\mathrm{H}, \mathrm{Cao} J$, Zhang $\mathrm{G}$, Wang Y. Kaempferol attenuates cardiac hypertrophy via regulation of ASK1/MAPK signaling pathway and oxidative stress. Planta Med. 2017;83(10):837-845.

15. Ren R, Chen SD, Fan J, Zhang G, Li JB. miRNA-138 regulates MLK3/JNK/ MAPK pathway to protect BV-2 cells from $\mathrm{H} 2 \mathrm{O} 2$-induced apoptosis. Bratisl Lek Listy. 2018;119(5):284-288.

16. Li Q, Park K, Xia Y, et al. Regulation of macrophage apoptosis and atherosclerosis by lipid-induced PKCdelta isoform activation. Circ Res. 2017;121(10):1153-1167.

17. van Diepen JA, Berbee JF, Havekes LM, Rensen PC. Interactions between inflammation and lipid metabolism: Relevance for efficacy of anti-inflammatory drugs in the treatment of atherosclerosis. Atherosclerosis. 2013;228(2):306-315.

18. Tabas I, Bornfeldt KE. Macrophage phenotype and function in different stages of atherosclerosis. Circ Res. 2016;118(4):653-667.

19. Zhang Q, Lenardo MJ, Baltimore D. 30 years of NF-kappaB: A blossoming of relevance to human pathobiology. Cell. 2017;168(1-2):37-57.

20. Liu Z, Gan L, Xu Y, et al. Melatonin alleviates inflammasome-induced pyroptosis through inhibiting NF-kappaB/GSDMD signal in mice adipose tissue. J Pineal Res. 2017;63(1). doi:10.1111/jpi.12414

21. Dong RF, Zhang B, Tai LW, Liu HM, Shi FK, Liu NN. The neuroprotective role of miR-124-3p in a 6-hydroxydopamine-induced cell model of Parkinson's disease via the regulation of ANAX5. J Cell Biochem. 2018;119(1):269-277.

22. de Ronde MWJ, KokMGM, Moerland PD, et al. High miR-124-3p expression identifies smoking individuals susceptible to atherosclerosis. Atherosclerosis. 2017;263:377-384.

23. Geng L, Liu W, Chen Y. miR-124-3p attenuates MPP(+)-induced neuronal injury by targeting STAT3 in SH-SY5Y cells. Exp Biol Med (Maywood). 2017;242(18):1757-1764.

24. Fan $X$, Wang $C$, Shi $P$, et al. Platelet MEKK3 regulates arterial thrombosis and myocardial infarct expansion in mice. Blood Adv. 2018;2(12): 1439-1448.

25. Ding SK, Wang LX, Guo LS, et al. Syringic acid inhibits apoptosis pathways via downregulation of p38MAPK and JNK signaling pathways in $\mathrm{H} 9 \mathrm{c} 2$ cardiomyocytes following hypoxia/reoxygenation injury. Mol Med Rep. 2017;16(2):2290-2294.

26. He H, Chang R, Zhang T, Yang C, Kong Z. ATM mediates DAB2IP-deficient bladder cancer cell resistance to ionizing radiation through the p38MAPK and NF-kappaB signaling pathway. MolMed Rep. 2017;16(2): 1216-1222.

27. Menon MB, Gropengiesser J, Fischer J, et al. p38(MAPK)/MK2-dependent phosphorylation controls cytotoxic RIPK1 signalling in inflammation and infection. Nat Cell Biol. 2017;19(10):1248-1259.

28. Cai Y, Li W, Tu H, et al. Curcumolide reduces diabetic retinal vascular leukostasis and leakage partly via inhibition of the p38MAPK/NFkappa B signaling. Bioorg Med Chem Lett. 2017;27(8):1835-1839.

29. Xu J, Tang S, Yin B, Sun J, Bao E. Co-enzyme Q10 upregulates Hsp70 and protects chicken primary myocardial cells under in vitro heat stress via PKC/MAPK. Mol Cell Biochem. 2018;449(1-2):195-206.

30. Cui M, Wang J, Li Q, Zhang J, Jia J, Zhan X. Long non-coding RNA HOXA11-AS functions as a competing endogenous RNA to regulate ROCK1 expression by sponging miR-124-3p in osteosarcoma. Biomed Pharmacother. 2017;92:437-444. 